University of Nebraska - Lincoln

DigitalCommons@University of Nebraska - Lincoln

Timothy J. Gay Publications

Research Papers in Physics and Astronomy

2013

\title{
Compact inline optical electron polarimeter
}

Munir Pirbhai

D. M. Ryan

G. Richards

Timothy J. Gay

Follow this and additional works at: https://digitalcommons.unl.edu/physicsgay

Part of the Atomic, Molecular and Optical Physics Commons, and the Other Physics Commons

This Article is brought to you for free and open access by the Research Papers in Physics and Astronomy at DigitalCommons@University of Nebraska - Lincoln. It has been accepted for inclusion in Timothy J. Gay Publications by an authorized administrator of DigitalCommons@University of Nebraska - Lincoln. 


\title{
Compact inline optical electron polarimeter
}

\author{
M. Pirbhai, D. M. Ryan, G. Richards, and T. J. Gay \\ Department of Physics and Astronomy, University of Nebraska, Lincoln, Nebraska 68588-0299, USA
}

(Received 19 February 2013; accepted 10 May 2013; published online 31 May 2013)

\begin{abstract}
A compact optical electron polarimeter using a helium target is described. It offers a maximum fluorescence detection efficiency of $\sim 20 \mathrm{~Hz} / \mathrm{nA}$, which is an order of magnitude higher than that of earlier designs. With an argon target, this device is expected to have a polarimetric figure-of-merit of $270 \mathrm{~Hz} / \mathrm{nA}$. By relying on a magnetic field to guide a longitudinally spin-polarized electron beam, the present instrument employs fewer electrodes. It also uses a commercially available integrated photon counting module. These features allow it to occupy a smaller volume and make it easier to operate. (C) 2013 AIP Publishing LLC. [http://dx.doi.org/10.1063/1.4807745]
\end{abstract}

\section{INTRODUCTION}

The scattering of spin-polarized electrons provides unique information about the dynamics of electron interactions with other particles, atoms, molecules, and surfaces. ${ }^{1-5}$ An ensemble of electrons is spin polarized if there exists an axis of quantization $\hat{\imath}$ along which the number of electrons with spin up, $N_{\uparrow}$, differs from that with spin down, $N_{\downarrow}$. The degree of polarization $P(\hat{\imath})$ of the ensemble is defined as $\left(N_{\uparrow}-N_{\downarrow}\right) /\left(N_{\uparrow}+N_{\downarrow}\right)$. If $P(\hat{\imath})$ is parallel or antiparallel to the electron momentum vector, the polarization is longitudinal, while for $P(\hat{\imath})$ perpendicular to the momentum vector, the particles are transversely polarized.

Several techniques have been devised to measure electron polarization. Mott polarimeters ${ }^{5-7}$ enjoy the widest application. These instruments measure the left-right scattering asymmetry resulting from the spin-orbit coupling when highenergy electrons are scattered at large angles from a high $\mathrm{Z}$ target. They offer good efficiencies and analyzing powers. However, they operate with high voltages $(20-100 \mathrm{kV})$, and only measure transverse polarization. Since most sources produce longitudinally polarized electrons, spin rotators are often required to create transversely polarized beams.

Optical electron polarimeters ${ }^{5,6,8-17}$ have no such constraints. In these devices, polarized electrons excite an atomic target, usually a noble gas, by exchange. The atom's spin-orbit coupling transfers the resulting atomic electron polarization to electronic orbital orientation. The atom emits circularly polarized fluorescence during the de-excitation process, and the electron polarization $P_{e}$ can be related to the relative Stokes parameter $P_{3}$, which describes the circular polarization of the light, by $P_{e}=P_{3} / A$. Here, $A$ refers to the analyzing power of the polarimeter. The analyzing power of optical electron polarimeters $(\sim 0.5-0.7)$ is generally higher than those of Mott polarimeters $(\sim 0.1-0.4)$. Moreover, it need not be measured. With the proper choice of atomic transitions and in the absence of cascade contributions to the fluorescence, the optical polarimeter's analyzing power can be calculated accurately from first principles. ${ }^{8,18}$

Inspired by recent efforts ${ }^{19-21}$ to decrease the size of Mott polarimeters, we have constructed a compact noble gas optical electron polarimeter. It improves on an earlier version ${ }^{22}$ reported by us. The current model uses fewer electrodes, relying on a uniform $10 \mathrm{mT}$ longitudinal magnetic field to guide the polarized electrons instead. Assuming helium targets, the present design offers figures-of-merit ${ }^{17}$ (FOM), which is inversely proportional to the square of the time required to make a measurement of $P_{e}$ to a given statistical uncertainty, $\sim 260$ times higher than the earlier version. It also occupies a quarter the volume. Unlike earlier helium optical electron polarimeters, ${ }^{10-15}$ which were designed for transversely polarized particles, this model can measure the polarization of longitudinally polarized beams.

In the optically pumped electron spin filter experiments ${ }^{23}$ for which this polarimeter has been developed, it has used helium as its target gas. Argon would provide $\sim 80$ times better figures-of-merit. ${ }^{22}$ However, its relevant atomic transition lies in the near-infrared region at $811 \mathrm{~nm}$, close to the wavelengths of lasers used in the rubidium optical pumping process $(795 \mathrm{~nm})$ for electron spin filters. This laser light might scatter in the polarimeter chamber and contaminate the signal of interest or even damage the photon counting module monitoring the atomic fluorescence.

The relative Stokes parameters $P_{1}, P_{2}$, and $P_{3}$ of the $388.9 \mathrm{~nm} 3^{3} \mathrm{P} \rightarrow 2^{3} \mathrm{~S}$ He radiation can be related analytically to the polarization of the electron beam $P_{e} \cdot{ }^{18} \mathrm{Here}, P_{1}, P_{2}$, and $P_{3}$ describe, respectively, the difference between the amount of horizontal and vertical linear polarization relative to the electron beam axis, of linear $+45^{\circ}$ or $-45^{\circ}$ polarization, and of right- or left-circular polarization of the light, all normalized to the total intensity. ${ }^{24}$ Applying the results of Bartschat et al. ${ }^{25}$ to our collision geometry, shown in Fig. 1, we find that

$$
P_{e}=P_{3}\left(\frac{2.6409}{1.0614+0.9386 P_{1}}\right) .
$$

Hence, by determining $P_{1}$ and $P_{3}$ experimentally, we can deduce the electron polarization. On the other hand, $P_{2}$ vanishes if the spin-orbit interaction in the Hamiltonian describing the electron-atom scattering is negligible, which is a necessary condition for the validity of Eq. (1). ${ }^{26}$ Therefore, measurement of $P_{2}=0$ serves as a check of the assumptions leading to Eq. (1). 


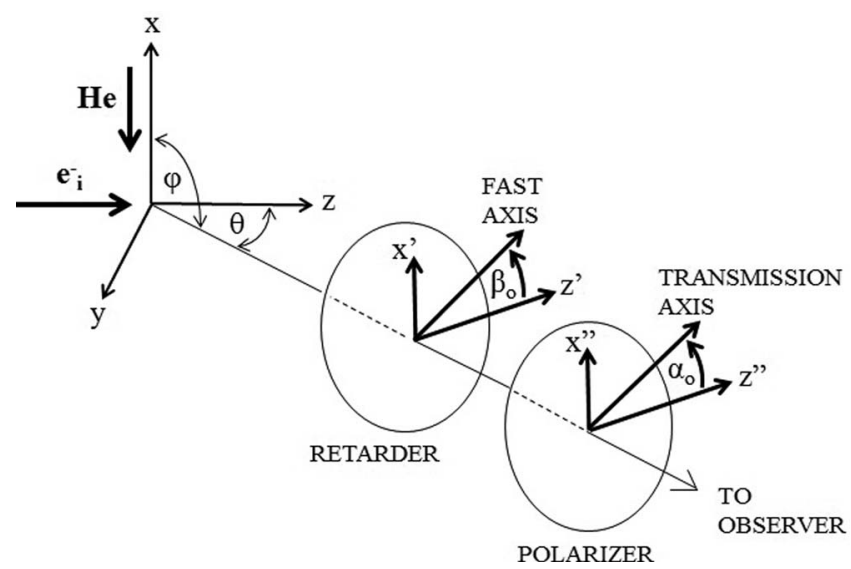

FIG. 1. Schematic of the He optical electron polarimeter geometry. In our device, $\varphi=90^{\circ}$ and $\theta=36.5^{\circ}$. The electron beam is incident along $\mathrm{z}$ while the helium gas flows towards the $-\mathrm{x}$ direction. The initial offsets of the retarder's fast axis and the linear polarizer's transmission axis are denoted by $\beta_{o}$ and $\alpha_{o}$, respectively.

\section{POLARIMETER DESIGN}

The electron polarimeter is shown in Figs. 2 and 3. The main vacuum chamber is constructed of aluminum. We used this metal because it is relatively easy to machine and it is non-magnetic. If, however, stray electrons strike the nonconductive oxide on an unprepared aluminum surface, they will charge the walls. ${ }^{27}$ To prevent this effect, we have coated the inside of this chamber with a thin, uniform layer of aqueous colloidal graphite $\left(\right.$ Aerodag $\left.^{\circledR}\right)$. The polarimeter main chamber contains eight Conflat ${ }^{\circledR}$ ports. The electron beam enters the chamber through the opening at A. The electron collector is located at the opposite end E. The latter consists of the halves of a longitudinally split, hollow cylindrical electrode. The halves are biased with potentials to deflect and collect the primary electron beam. The polarimeter is currently being used at the downstream end of an electron spin-filter, ${ }^{23}$ and the $795 \mathrm{~nm}, 0.6 \mathrm{~W}$ optical pumping laser enters the system through the anterior viewport of the electron collector, counter propagating with the electron beam. A pressure gauge

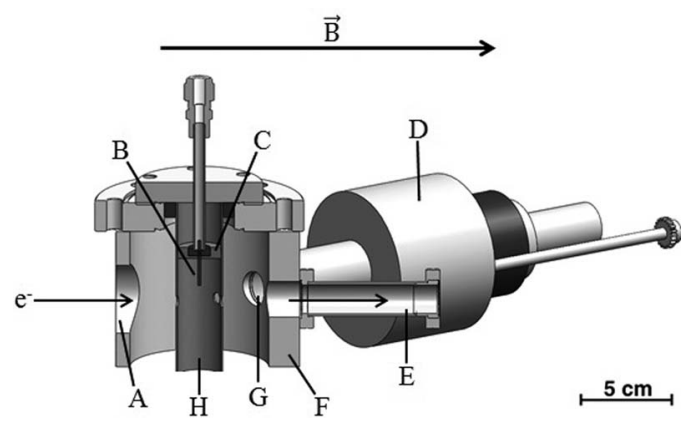

FIG. 2. Vertical cut-away view of the electron polarimeter. The incident electrons enter the chamber through A traveling from left-to-right, guided by a longitudinal $10 \mathrm{mT}$ magnetic field. Shown also are the target-gas-feed copper capillary $\mathrm{B}$, its mounting sleeve $\mathrm{C}$, the optical polarimeter $\mathrm{D}$, the chamber housing the electron collector and viewport $\mathrm{E}$, the main vacuum chamber F, the fluorescence collection lens G, and the stainless steel inner energydefining cylinder $\mathrm{H}$.

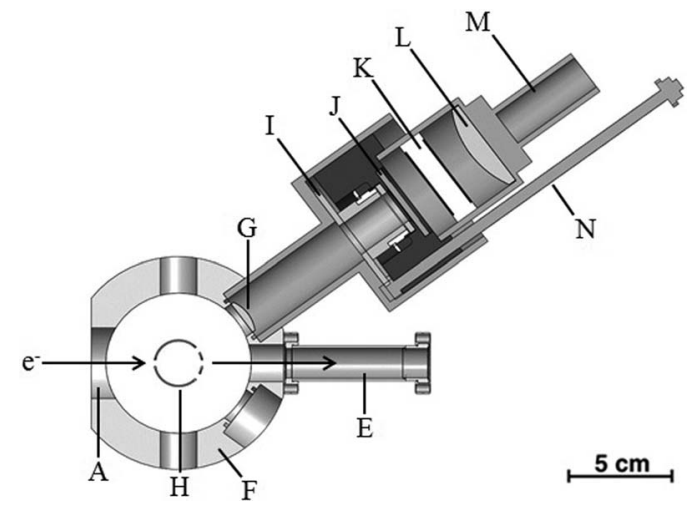

FIG. 3. Horizontal cross-sectional view of the optical polarimeter. Letter designations for the collision chamber are the same as those in Fig. 2. Shown also are the hollow gear shaft on which the retarder is mounted I, the linear polarizer $\mathrm{J}$, the interference filter $\mathrm{K}$, the focusing lens $\mathrm{L}$, and the photon counting module $\mathrm{M}$. The rod $\mathrm{N}$ has step-down gears at either end and connects the retarder to a stepper motor.

is mounted on one of the ports. A $60 \mathrm{~L} / \mathrm{s}$ turbomolecular pump (Pfeiffer Vacuum HiPace 80), attached at the bottom of the chamber, maintains the vacuum at a base pressure of $10^{-7}$ Torr, which rises to $10^{-4}$ Torr when gas is introduced.

The helium injection assembly is fitted to the top of the polarimeter chamber. Target gas flows through a feedthrough, with an outer diameter of 0.25 in., welded on a 2.75 in. Conflat ${ }^{\circledR}$ flange before passing into a copper capillary with 0.125 in. outer and 0.061 in. inner diameters. The end of the capillary is $\sim 5 \mathrm{~mm}$ above the electron beam. Trantham et al. ${ }^{16}$ have shown that the fluorescence intensity is close to a maximum at this height while, Fischer and Kessler ${ }^{12}$ have demonstrated that the Stokes parameters are independent of height above this value. A stainless steel cylinder of outer diameter $25.5 \mathrm{~mm}$ and wall thickness $1 \mathrm{~mm}$ encloses the helium injection assembly. The former is electrically isolated from the rest of the chamber. It is used as an electrode to define the nominal electrical potential of the collision volume, and also serves to direct the effusive flow of gas from the target capillary into the turbo pump.

The metallic inner energy-defining cylinder has two collinear apertures (Fig. 3), each $6 \mathrm{~mm}$ in diameter, through which the electron beam enters and exits the collision region. Photons emitted from the excited helium pass through a $40 \mathrm{~mm}$ focal-length collection lens, and travel to the optical train via an opening at $36.5^{\circ}$ from the electron beam axis. For maximum efficiency in measuring longitudinal polarization, the optical polarimeter should be located on axis with the electron propagation direction. However, we had to leave room for the chamber housing the Faraday cup, and more importantly, for the window through which the laser used in the optical pumping process enters the apparatus. Therefore, the minimum angle at which the optical polarimeter could be positioned was $36.5^{\circ}$.

For a given $P_{e}$, the relative Stokes parameters $P_{1}$ and $P_{3}$ are functions of electron energy. Therefore, to obtain $P_{e}$ accurately, the energy spread of the beam, and hence, the electric potential must vary minimally in the region yielding detectable fluorescence from the excitation of the target gas by 


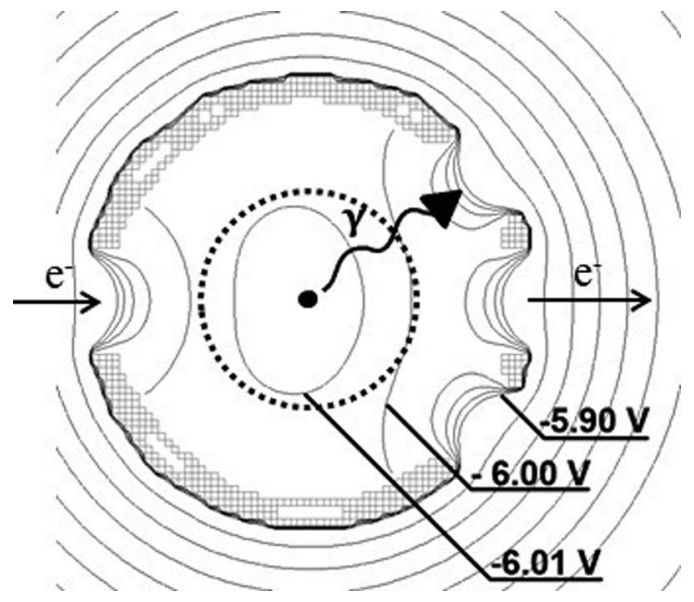

FIG. 4. SIMION 8.0 simulation of the contour lines inside the stainless steel inner energy-defining cylinder. A horizontal slice through the center is shown. The potential varies by less than $0.3 \%$ along the path of the electron beam in the region where the helium atoms are excited. Here, the focal point of the fluorescence collection lens is indicated (-), while the region within the dashed circle represents the "sensitive volume" 28 of the optical polarimeter.

the electrons. To provide an electric potential as uniform as possible in this region, the capillary should be at the same voltage as the stainless steel cylinder. Therefore, it cannot be welded to the feedthrough, which is grounded. It is instead fitted through a cylindrical Delrin ${ }^{\circledR}$ retainer which is surrounded by a copper mounting sleeve. The sleeve is in electrical contact with the outer metallic cylinder as well as with the copper capillary. For the optically pumped electron spin filter experiments, $\mathrm{a}-6 \mathrm{~V}$ potential is applied to the cylinder when the relative Stokes parameters are measured at electron energies corresponding to the peak of the helium $2^{3} \mathrm{~S} \rightarrow 3^{3} \mathrm{P}$ optical excitation function. The outer vacuum chamber is held at ground. While the electric potential may vary spatially in the collision volume due to contact potential differences between the cylinder and the capillary, a SIMION ${ }^{\circledR}$ simulation, shown in Fig. 4, revealed that the electric potential changes by less than $0.3 \%$ within this region. Here, a contact potential of $-0.5 \mathrm{~V}$ for the capillary relative to the inner cylinder was assumed. Indeed, data near the threshold of the helium $2^{3} \mathrm{~S}$ $\rightarrow 3^{3} \mathrm{P}$ optical excitation function are consistent with no energy broadening due to spatial variations of the electric potential in the vicinity of the capillary tip.

The optical polarimeter, shown in Fig. 3, uses a rotating retarder and a fixed linear polarizer downstream to determine the relative Stokes parameters $P_{1}, P_{2}$, and $P_{3}$. Measurements of $P_{e}$ are, therefore, unaffected by polarization sensitivity of the photon detector because it observes a single polarization of light. The optical train comprises a collimating lens, the retarder, linear polarizer, an interference filter, a focusing lens, and a photon counting module. The retarder and linear polarizer are cut from plastic sheets (International Polarizer Inc. No. IP160WR-P and Rolyn No. 65.5305, respectively) commonly available in student laboratories. The retarder is mounted on a hollow gear shaft. The latter is connected to a stepper motor via a rod with step-down gears at either end. The stepper motor can, thus, rotate the retarder in increments of $7.5^{\circ}$. The photodetector is a Hamamatsu photon counting head (H6180-01), which includes a bialkali photomultiplier tube, a high-voltage dc/dc power supply, and a high-speed photon counting circuit integrated into a compact unit, which minimizes the electron polarimeter's footprint. Furthermore, it only requires connecting a $+5 \mathrm{Vdc}$ power supply and a pulse counter. It is straightforward to operate because there is no need for setting discrimination levels or high voltage adjustment.

The entire device occupies a relatively small volume, $\sim 0.03 \mathrm{~m}^{3}$. It can easily be dismounted and moved to other experiments. LabView ${ }^{\circledR}$ software fully automates its operation controlling the rotation of the stepper motor, the application of voltages to the inner cylinder, and the acquisition of data related to photon count rate, Faraday cup current, and pressure in the chamber.

\section{OPERATION}

Evaluation of the relative Stokes parameters consists of first rotating the retarder incrementally by $22.5^{\circ}$ for one revolution, and measuring the intensity of the fluorescence at each position of the fast axis. The pressure in the chamber and the current on the Faraday cup are recorded at each retarder position. This procedure is repeated for electron energies both below and above that corresponding to the onset of the transition. The below-threshold unnormalized intensity is subtracted from the threshold value to eliminate the effect of background light on the measurement. The result is then normalized to the chamber pressure and Faraday cup current. ${ }^{22}$

According to the Mueller calculus, the normalized transmitted intensity $I_{i}^{\prime}$ for light incident on such an optical polarimeter obeys ${ }^{15}$

$$
\begin{aligned}
I_{i}^{\prime}= & I\left\{1+\frac{1}{2} P_{1} k_{\text {inc }}\left\lfloor\cos \left(2 \alpha_{o}\right)\left[\cos \left(4 \beta_{i}+4 \beta_{o}\right)(1-\cos \delta)+(1+\cos \delta)\right]+\sin \left(2 \alpha_{o}\right) \sin \left(4 \beta_{i}+4 \beta_{o}\right)(1-\cos \delta)\right\rfloor\right. \\
& +\frac{1}{2} P_{2} k_{i n c}\left\lfloor\cos \left(2 \alpha_{o}\right) \sin \left(4 \beta_{i}+4 \beta_{o}\right)(1-\cos \delta)-\sin \left(2 \alpha_{o}\right)\left[\cos \left(4 \beta_{i}+4 \beta_{o}\right)(1-\cos \delta)-(1+\cos \delta)\right]\right\rfloor \\
& \left.-P_{3} k_{i n c} \sin \left(2 \beta_{i}+2 \beta_{o}-2 \alpha_{o}\right) \sin \delta\right\} .
\end{aligned}
$$




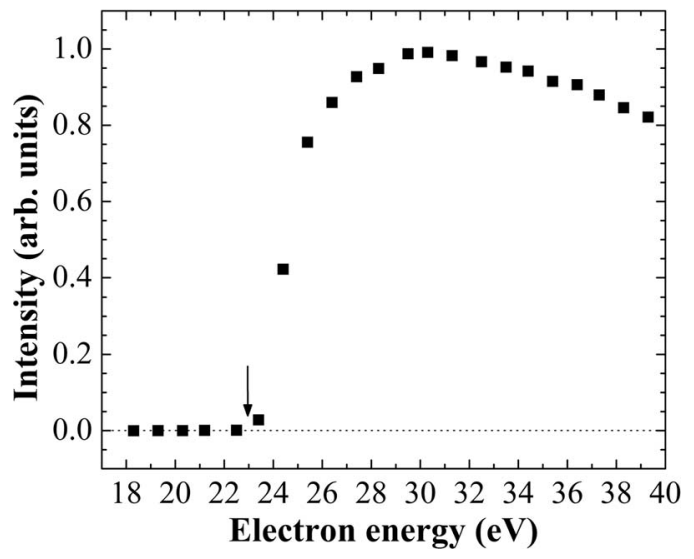

FIG. 5. Excitation function for the transition of helium $2^{3} \mathrm{~S} \rightarrow 3^{3} \mathrm{P}$, $388.9 \mathrm{~nm}$. Arrow indicates the known threshold energy.

Here, $\delta$ is the retardance of the waveplate, and $k_{i n c}$ is a measure of the efficiency ${ }^{24}$ of the linear polarizer. The latter is given by $\left(k_{1}-k_{2}\right) /\left(k_{1}+k_{2}\right)$, where $k_{1}$ and $k_{2}$ are the maximum and minimum transmittances of completely linearly polarized light through the polarizer. The retarder angle $\beta_{i}$ is equal to $(\mathrm{i}-1) \times 22.5^{\circ}$, where $\mathrm{i}=1,2, \ldots 16$.

As noted by Berry et al. ${ }^{24}$ Eq. (2) describes a truncated Fourier series. Therefore, the relative Stokes parameters can be obtained in terms of Fourier coefficients as outlined in Refs. 15 and 24. We analyze the polarimetric data, and compute $P_{1}, P_{2}$, and $P_{3}$ by following the algorithm described in these references. Comprehensive accounts of how to calibrate $\delta, k_{\text {inc }}, \alpha_{o}$, and $\beta_{o}$ also exist in the literature. ${ }^{10,15,16,24} \mathrm{We}$ obtained the following for our experimental setup: $\delta=(1.65$ $\pm 0.01)$ radians, $k_{i n c}=(0.971 \pm 0.001), \alpha_{o}=(0 \pm 1)^{\circ}$, and $\beta_{o}=(35 \pm 1)^{\circ}$.

Equation (1) for $P_{e}$ is valid for electron energies between the threshold energy for the helium $3^{3} \mathrm{P}$ excitation, at $23.0 \mathrm{eV}$, and that corresponding to the first cascading transition $4^{3} \mathrm{~S}$ $\rightarrow 3^{3} \mathrm{P}$, a range of $\sim 0.6 \mathrm{eV}$. Due to contact potential differences, the electron energy is not necessarily the electric charge times the applied difference in potential between the target and the electron emitter. Therefore, the first step in a complete polarimetric measurement involves determining the energy of the beam. The energy scale is established by measuring the optical excitation function for the helium $3^{3} \mathrm{P}$ state. Figure 5 shows such an optical excitation function. The arrow indicates the onset of the signal of interest above background. For better counting statistics, $P_{3}$ can be measured at electron energies corresponding to the peak of the optical excitation function. The values will differ from those obtained at threshold due to cascading effects. ${ }^{12}$ The electron polarization is then determined by calibrating the measurements with those made between 23.0 and $23.6 \mathrm{eV}$.

The value of $P_{1}$ needed in Eq. (1) can be determined using unpolarized electrons. Such data, taken at $23.6 \mathrm{eV}$, are shown in Fig. 6. The fast axis of the retarder was rotated incrementally by $7.5^{\circ}$, and the fluorescence intensity was measured at each position. The error bars represent the standard deviation of the mean of five datasets taken through five complete retarder rotations. A value of $P_{1}=0.026 \pm 0.005$ is

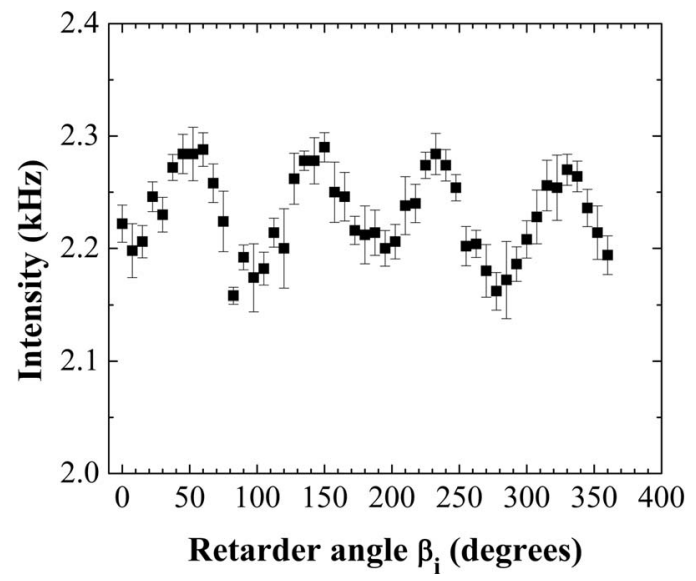

FIG. 6. Variation in fluorescence intensity as the retarder is rotated. The electron beam energy is $23.6 \mathrm{eV}$.

extracted from these results. From Fig. 6, we also determine that the efficiency of the polarimeter, $\eta$, is about $2.3 \mathrm{~Hz} / \mathrm{nA}$ for a chamber pressure of $1 \mathrm{mTorr}$, as read by the Convectron ${ }^{\circledR}$ gauge corrected for helium. Hence, the FOM, $\eta A^{2},{ }^{17}$ is $\sim 0.39 \mathrm{~Hz} / \mathrm{nA}$ at this energy. The efficiency rises to $\sim 20 \mathrm{~Hz} / \mathrm{nA}$ for electron energies corresponding to the peak of the optical excitation function, yielding a FOM of $3.4 \mathrm{~Hz} / \mathrm{nA}$. By comparison, our earlier model ${ }^{22}$ would provide a FOM a factor of $\sim 260$ lower than these values using a helium target. An argon target would increase the FOM of the current design to about $270 \mathrm{~Hz} / \mathrm{nA}$. Given this value and a beam current of $1 \mu \mathrm{A}$, the polarimeter would take $\sim 1 / 2 \mathrm{~s}$ of photon counting time to determine an electron polarization of $20 \%$ to within $1 \%$ of itself, based on counting statistics alone. If the efficiency is redefined as the number of photon counts per incident electron (a more standard definition), the device's maximum FOM, with argon, is $\sim 10^{-8}$, as compared with $\sim 10^{-4}$ for micro-Mott polarimeters. ${ }^{6,7,19-21}$

Figure 7 shows how $P_{1}$ and $P_{2}$ vary as the energy of the electron beam changes from approximately $23.1 \mathrm{eV}$ to $30.1 \mathrm{eV}$. The values of $P_{2}$ are zero within the experimental uncertainty as expected. The $P_{1}$ parameter shows its characteristic minimum ${ }^{11-13}$ around $24.5 \mathrm{eV}$.

The $3^{3} \mathrm{P}$ state can only be excited from the singlet ground state of helium through the electron exchange interaction.

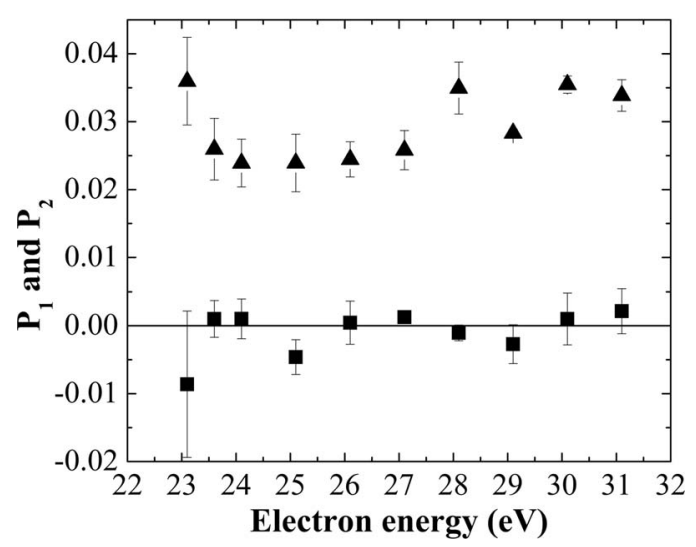

FIG. 7. Energy dependence of $P_{1}(\boldsymbol{\Lambda})$ and $P_{2}(\mathbf{\square})$. 


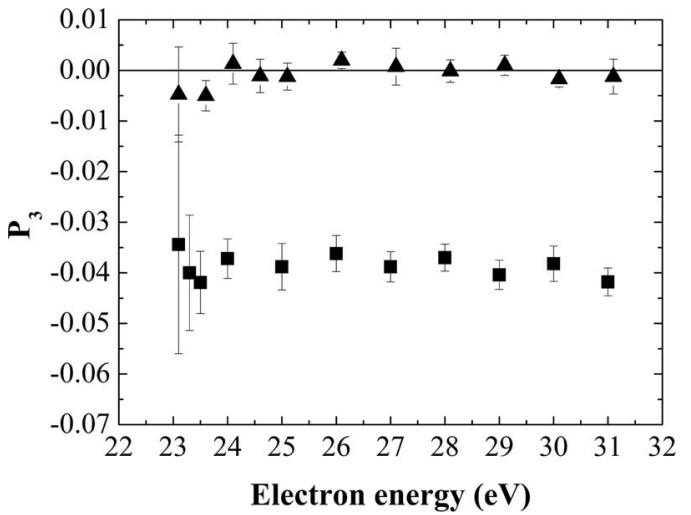

FIG. 8. Energy dependence of $P_{3}$ with unpolarized $(\boldsymbol{\Lambda})$ and polarized electrons.

Figure 8 illustrates the importance of this interaction. The relative circular polarization of the helium $3^{3} \mathrm{P} \rightarrow 2^{3} \mathrm{~S}$ fluorescence is zero within the experimental uncertainty for unpolarized electrons and $(-4.1 \pm 0.5) \%$ for polarized electrons from our source. Applying Eq. (1) to the $P_{3}$ data within the energy range where it is valid, we obtain an electron polarization of $(-10 \pm 1) \%$. The uncertainty was determined by propagating the errors in $P_{1}$ and $P_{3}$.

In conclusion, we have constructed and tested a compact helium electron polarimeter. This instrument offers several attractive features for electron spin analysis. It is relatively simple in its construction and is compact. Unlike previous helium electron polarimeters, it can measure the polarization of longitudinally polarized electrons. It also provides $\sim 260$ times higher figures-of-merit than earlier versions we have reported. An argon target would raise this figure-of-merit by a factor of $\sim 80 .^{22}$

\section{ACKNOWLEDGMENTS}

The authors gratefully acknowledge useful discussions with J. W. Maseberg and E. T. Litaker. M.P. wishes to thank M. Bradaric for her assistance with generating the figures. This work was supported by National Science Foundation (NSF) Grant Nos. PHY-0855629 and PHY-0821385.
${ }^{1}$ J. Kessler, Polarized Electrons, 2nd ed. (Springer, Berlin, 1985).

${ }^{2}$ Polarized Electrons in Surface Physics, edited by R. Feder (World Scientific, Singapore, 1985).

${ }^{3}$ D. A. Tulchinsky, M. H. Kelley, J. J. McClelland, R. Gupta, and R. J. Celotta, J. Vac. Sci. Technol. A 16, 1817 (1998).

${ }^{4}$ J. A. Borchers, J. A. Dura, J. Unguris, D. Tulchinsky, M. H. Kelley, C. F. Majkrzak, S. Y. Hsu, R. Loloee, W. P. Pratt, Jr., and J. Bass, Phys. Rev. Lett. 82, 2796 (1999).

${ }^{5}$ T. J. Gay, Advances in Atomic, Molecular and Optical Physics (Academic Press, Burlington, MA, 2009), Vol. 57, Chap. 4.

${ }^{6}$ T. J. Gay, in Atomic, Molecular, and Optical Physics: Charged Particles, edited by F. B. Dunning and R. G. Hulet (Academic, New York, 1995), Vol. 29A.

${ }^{7}$ T. J. Gay and F. B. Dunning, Rev. Sci. Instrum. 63, 1635 (1992).

${ }^{8}$ M. Eminyan and G. Lampel, Phys. Rev. Lett. 45, 1171-1174 (1980).

${ }^{9}$ B. Collin, J. Arianer, S. Essabaa, R. Frascaria, R. Gacougnolle, R. Kunne, K. Aulenbacher, and V. Tioukine, Nucl. Instrum. Methods Phys. Res. A 534, 361 (2004).

${ }^{10}$ A. B. Wedding, A. G. Mikosza, and J. F. Williams, J. Opt. Soc. Am. A 8, 1729 (1991).

${ }^{11}$ I. Humphrey, C. Ranganathaiah, J. L. Robins, J. F. Williams, R. A. Anderson, and W. C. Macklin, Meas. Sci. Technol. 3, 884 (1992).

${ }^{12}$ T. Fischer and J. Kessler, Rev. Sci. Instrum. 66, 4885 (1995).

${ }^{13}$ P. A. Hayes, D. H. Yu, J. Furst, M. Donath, and J. F. Williams, J. Phys. B 29, 3989 (1996).

${ }^{14}$ R. Cun-Jun, P. Wen-Ning, G. Jun-Fang, and S. Ren-Cheng, Chin. Phys. 11, 126 (2002).

${ }^{15}$ J. W. Maseberg and T. J. Gay, J. Phys. B 39, 4861 (2006).

${ }^{16}$ K. W. Trantham, T. J. Gay, and R. J. Vandiver, Rev. Sci. Instrum. 67, 4103 (1996).

${ }^{17}$ T. J. Gay, J. E. Furst, K. W. Trantham, and W. M. K. P. Wijayaratna, Phys. Rev. A 53, 1623-1629 (1996).

${ }^{18}$ T. J. Gay, J. Phys. B 16, L553 (1983).

${ }^{19}$ G. C. Burnett, T. J. Monroe, and F. B. Dunning, Rev. Sci. Instrum. 65, 1893 (1994).

${ }^{20}$ G. Ghiringhelli, K. Larsson, and N. B. Brooks, Rev. Sci. Instrum. 70, 4225 (1999).

${ }^{21}$ D. D. Neufeld, H. Aliabadi, and F. B. Dunning, Rev. Sci. Instrum. 78, 025107 (2007).

${ }^{22}$ J. E. Furst, W. M. K. P. Wijayaratna, D. H. Madison, and T. J. Gay, Phys. Rev. A 47, 3775-3787 (1993).

${ }^{23}$ H. Batelaan, A. S. Green, B. A. Hitt, and T. J. Gay, Phys. Rev. Lett. 82, 4216-4219 (1999).

${ }^{24}$ H. G. Berry, G. Gabrielse, and A. E. Livingston, Appl. Opt. 16, 3200 (1977).

${ }^{25}$ K. Bartschat, K. Blum, G. F. Hanne, and J. Kessler, J. Phys. B 14, 3761 (1981).

${ }^{26}$ K. Bartschat and K. Blum, Z. Phys. A 304, 85 (1982).

${ }^{27}$ T. G. Anderson, B. G. Birdsey, S. M. Woeher, M. A. Rosenberry, and T. J. Gay, Rev. Sci. Instrum. 72, 2923 (2001).

${ }^{28}$ E. T. Litaker, J. R. Machacek, and T. J. Gay, Eur. J. Phys. 32, 1107 (2011). 